\title{
Distribution kinetics of polymer crystallization and the Avrami equation
}

\author{
Jiao Yang and Benjamin J. McCoy \\ Department of Chemical Engineering, Louisiana State University, Baton Rouge, Louisiana 70803 \\ Giridhar Madras ${ }^{\text {a) }}$ \\ Department of Chemical Engineering, Indian Institute of Science, Bangalore 560 012, India
}

\begin{abstract}
Cluster distribution kinetics is adopted to explore the kinetics of polymer crystallization. Population balance equations based on crystal size distribution and concentration of amorphous polymer segments are solved numerically and the related dynamic moment equations are also solved. The model accounts for heterogeneous or homogeneous nucleation and crystal growth. Homogeneous nucleation rates follow the classical surface-energy nucleation theory. Different mass dependences of growth and dissociation rate coefficients are proposed to investigate the fundamental features of nucleation and crystal growth. A comparison of moment solutions with numerical solutions examines the validity of the model. The proposed distribution kinetics model provides a different interpretation of the familiar Avrami equation.
\end{abstract}

\section{INTRODUCTION}

Since the discovery of crystallization of thin lamellar polymer crystals in solution, ${ }^{1}$ the study of polymer crystallization has received considerable attention. Polymer crystallization controls the macroscopic structure of the material, and thereby determines the properties of final polymer products. $^{2,3}$ The morphology of polymer crystals is different from that of crystals consisting of simple molecules, mainly due to the difference between the chain connectivity in polymers and the assemblies of simple molecules. ${ }^{4}$ This not only affects the equilibrium crystal structures but also the kinetics of crystal growth. When the system is cooled from the equilibrium melting temperature $T_{m}$ to a lower crystallization temperature, the polymer crystals can form two-dimensional (2D) lamellar structures in both melt and solution ${ }^{5}$ via the stages: nucleation, lamellae growth, and spherulite aggregative growth. ${ }^{6}$ The formation of three-dimensional crystal structure from a disordered state begins with nucleation and involves the creation of a stable nucleus from the disordered polymer melt or solution. ${ }^{7}$ Depending on whether any second phase, such as a foreign particle or surface from another polymer, is present in the system, the nucleation is classified as homogenous nucleation (primary nucleation) or heterogeneous nucleation (secondary nucleation). ${ }^{8}$ In primary nucleation, creation of the stable nucleus by intermolecular forces orders the chains in a parallel array. As the temperature goes below the melting temperature $T_{m}$, the molecules tend to move toward their lowest energy conformation, a stiffer chain segment, and this will favor the formation of ordered chains and thus nuclei. Facilitating the formation of stable nuclei, secondary nucleation is also involved at the beginning of crystallization through heterogeneous nucleation agents, such as dust particles. Following nucleation, crystals

\footnotetext{
a) Author to whom correspondence should be addressed. Fax: 91-080-23600683. Electronic mail: giridhar@chemeng.iisc.ernet.in
}

grow by the deposition of chain segments on the nucleus surface. This growth is controlled by a small diffusion coefficient at low temperature and by thermal redispersion of chains at the crystal/melt interface at high temperature. ${ }^{9}$ Thus crystallization can occur only in a range of temperatures between the glass transition temperature $T_{g}$ and the melting point $T_{m}$, which is always higher than $T_{g}$.

As a consequence of their long-chain nature, subsequent entanglements, and particular crystal structure, polymers crystallized in the bulk state are never totally crystalline and a fraction of the polymer is amorphous. Polymers fail to achieve complete crystallinity because polymer chains cannot completely disentangle and align properly during a finite period of cooling. Lamellar structures can be formed, but a single polymer chain may pass through several lamellae with the result that some segments of the polymer chain are crystallized into the lamellae and some parts of the polymer chain are in the amorphous state between adjacent lamellae.

A well-known description of crystallization kinetics is the heuristic Avrami phase transition theory. Based on work of Avrami, ${ }^{10}$ who adapted the formulations intended for metallurgy to the needs of polymer crystallization, the original derivations were simplified by Evans ${ }^{11}$ and rearranged for polymer crystallization by Meares ${ }^{12}$ and Hay. ${ }^{13}$ For the bulk crystallization of polymers, the crystallization kinetics can be represented as

$$
1-X=e^{-V_{t}}
$$

where $X$ is the degree of crystallization and $V_{t}$ is the volume of crystallization material, which should be determined by considering the following two cases: (a) the nuclei are predetermined, that is, they all develop at once on cooling the polymer, and (b) the crystals nucleate sporadically. For a spherical crystal in case (a), 


$$
d V_{t}=4 \pi r^{2} L d r,
$$

where $r$ represents the radius of the spherical crystal at time $t$ and $L$ is the number of nuclei. Assuming the radius grows linearly with time, $r=\kappa t$, upon integration of Eq. (1.2) and substitution into Eq. (1.1), one obtains

$$
1-X=e^{-K t^{3}},
$$

where $K=(4 / 3) \pi \kappa^{3} L$ is the growth rate. For sporadic nucleation, case (b), the above argument is followed, but the number of spherical nuclei is allowed to increase linearly with time at rate $u$. Then nucleation from time $t_{i}$ to time $t$ will create a volume increase of

$$
d V_{t}=(4 / 3) \pi \kappa^{3}\left(t-t_{i}\right)^{3} u d t_{i} .
$$

Upon integration of Eq. (1.4) between $t_{i}=0$ and $t$, and substitution into Eq. (1.1), one obtains

$$
1-X=e^{-K t^{4}},
$$

where $K=(1 / 3) \pi \kappa^{3} u$. The equations can be generalized by replacing the power of $t$ with the Avrami exponent $n$,

$$
1-X=e^{-K t^{n}} .
$$

Thus, according to these arguments, the Avrami exponent $n$ depends not only on the structure of the crystal but also on the nature of nucleation. ${ }^{14}$

Though numerous models of crystal growth kinetics have been developed, ${ }^{15}$ the Avrami equation with its basis in rather empirical ideas is still applied to polymer crystallization. Our aim is to investigate if the Avrami equation can be established by a more fundamental approach to crystallization that incorporates homogeneous and heterogeneous nucleation, uneven growth of crystals into a particle size distribution, and final Ostwald ripening of the crystal size distribution. The distribution kinetics model ${ }^{16,17}$ of nucleation, growth, and aggregation results in an $S$-shape curve of crystallinity versus time. Considering the deposition of polymer chain on a crystal surface is similar to monomer attachment on a cluster, we adapt this kinetics model to explore polymer crystallization. An advantage of this model is the representation by rate coefficients of the microscopic polymer crystallization kinetics, making the model straightforward to understand, yet based on modern molecular concepts. To examine the validity of this model, we will compare the results with the Avrami equation ${ }^{18}$ and also relate the parameters of the two models.

\section{DISTRIBUTION KINETICS OF POLYMER CRYSTALLIZATION}

Homogenous nucleation can occur when the solution is supersaturated and thus metastable. Because of the great increase of the colliding probability among solute molecules in supersaturated solution, density fluctuations increase in intensity and frequency allowing nuclei to form sporadically. Classical homogeneous nucleation in the capillarity approximation ${ }^{19}$ is based on the sum of surface energy and formation free energy for a spherical cluster of radius $r$,

$$
W(r)=4 \pi r^{2} \sigma-(4 / 3) \pi r^{3}\left(\rho / x_{m}\right) k_{B} T \ln S .
$$

Here, $\sigma$ is the crystal interfacial energy and $\Delta G=-k_{B} T \ln S$ is the chemical potential difference between the two phases (the polymer solution or melt and crystal phase) in terms of supersaturation $S$. The typical structure of polymer crystal is thin lamellae and because of the equal probability of deposition in the two lateral directions, an equilateral lamellar structure is proposed. The total energy of such a 2D lamellar crystal is presented as

$$
W(a)=4 a L \sigma-a^{2} L\left(\rho / x_{m}\right) k_{B} T \ln S,
$$

where $a$ is the lateral length and $L$ is the thickness of the lamellae. Obviously, the energy $W(a)$ of a crystal increases with $a$ and then decreases from the maximum value $W^{*}$ at the critical lamellar length,

$$
a^{*}=2 \sigma x_{m} /\left(\rho k_{B} T \ln S\right) .
$$

Thus the maximum energy of the crystal, by replacing $S$ with $m^{(0)} / m_{\infty}^{(0)}$ according to the definition of supersaturation, is represented as

$$
W^{*}=4 x_{m} L \sigma^{2} /\left[\rho k_{B} T \ln \left(m^{(0)} / m_{\infty}^{(0)}\right)\right] .
$$

Here the local-equilibrium concentration is $m_{\mathrm{eq}}^{(0)}$ and the solubility of a flat surface is $m_{\infty}^{(0)}$. The expression for the nucleation rate $\mathrm{e}^{20}$ is derived from the flux over the energy barrier at the critical nucleus size,

$$
I=k_{n} \exp \left(-W^{*} / k_{B} T\right)
$$

with prefactor

$$
k_{n}=\left(m^{(0)}\right)^{2}\left(2 \sigma x_{m} / \pi\right)^{1 / 2} \rho^{-1}
$$

written in terms of monomer concentration $m^{(0)}$ and crystal density $\rho$.

For a crystal with curved surface, the local-equilibrium interfacial concentration at the crystal surface $m_{\mathrm{eq}}^{(0)}$ is related to the solubility of a flat surface $m_{\infty}^{(0)}$ by the Gibbs-Thomson equation,

$$
m_{\mathrm{eq}}^{(0)}=m_{\infty}^{(0)} \exp (\Omega),
$$

where $\Omega=2 \sigma x_{m} / r \rho k_{B} T$ in terms of monomer molecular mass $x_{m}$, surface energy $\sigma$, radius of curvature $r$, Boltzmann constant $k_{B}$, and absolute temperature $T$. For a $2 \mathrm{D}$ crystal lamella, however, the growth front is a flat surface and the radius of curvature $r$ is infinite. Thus, consistent with Eq. (2.7), the difference between local-equilibrium concentration $m_{\mathrm{eq}}^{(0)}$ and the solubility of a flat surface $m_{\infty}^{(0)}$ is negligible because $\Omega$ vanishes as $r$ approaches infinity.

The crystal mass distribution is defined so that $c(x, t) d x$ represents the molar concentration of crystals having values of mass $x$ in the range of $x$ to $x+d x$ at time $t$. Integral forms of the rate expressions in the population balance equation lead to moment calculations of the crystals and monomers. The general moments are defined as integrals of the crystal distribution over $x$, 


$$
c^{(n)}(t)=\int_{0}^{\infty} c(x, t) x^{n} d x .
$$

The zeroth moment $(n=0)$ is the total number (or concentration) of crystals; the first moment stands for the mass concentration of the crystals. The average crystal mass is the ratio of first moment over zeroth moment, $c^{\text {avg }}(t)$ $=c^{(1)}(t) / c^{(0)}(t)$. The monomers are assumed monodisperse with moments $m^{(n)}(t)=x_{m}^{n} m^{(0)}(t)$.

Similar to cluster growth in the distribution kinetics model, ${ }^{20}$ crystallization is the gradual building up of monomer on the nucleus surface in a melt or solution. A general representation of chain deposition on the crystal surface is

$$
C(x)+M\left(x_{m}\right) \underset{k_{d}}{\stackrel{k_{g}}{\rightleftharpoons}} C\left(x+x_{m}\right) .
$$

The rate coefficients $k_{g}$ and $k_{d}$ are for growth and dissociation, respectively. Different from general cluster distribution theory, crystal breakage and aggregation are usually not considered in polymer crystallization.

The population balance equations ${ }^{21}$ that govern the distributions of crystals and monomer are

$$
\begin{aligned}
\partial c(x, t) / \partial t= & -k_{d} c(x, t)+k_{d} \int_{x}^{\infty} c\left(x^{\prime}, t\right) \delta\left[x-\left(x^{\prime}-x_{m}\right)\right] d x^{\prime} \\
& -k_{g} c(x, t) \int_{0}^{\infty} m^{(0)} \delta\left(x^{\prime}-x_{m}\right) d x^{\prime} \\
& +k_{g} m^{(0)} \int_{0}^{x} c\left(x^{\prime}, t\right) \delta\left(x-x_{m}\right) d x^{\prime}+I \delta\left(x-x^{*}\right)
\end{aligned}
$$

and

$$
\begin{aligned}
\partial m(x, t) / \partial t= & -k_{g} m^{(0)} \int_{0}^{\infty} c\left(x^{\prime}, t\right) d x^{\prime}+k_{d} \int_{x}^{\infty} c\left(x^{\prime}, t\right) \\
& \times \delta\left(x-x_{m}\right) d x^{\prime}-I \delta\left(x-x^{*}\right) x^{*} / x_{m},
\end{aligned}
$$

where the homogeneous nucleation rate for crystals of critical nucleus mass $x^{*}$ is $I \delta\left(x-x^{*}\right)$. The distribution of the crystals changes according to Eq. (2.10), which becomes, when the integrations over the Dirac distributions are performed, the finite-difference differential equation,

$$
\begin{aligned}
\partial c(x, t) / \partial t= & -k_{d} c(x, t)+k_{d} c\left(x+x_{m}\right)-k_{g} c(x) m^{(0)} \\
& +k_{g} c\left(x-x_{m}\right) m^{(0)}+I \delta\left(x-x^{*}\right) .
\end{aligned}
$$

\section{A. Moment methods}

The general moment equations are determined by applying the operation $\int_{0}^{\infty}[] x^{n} d x$ to Eqs. (2.10) and (2.11), which yields

$$
\begin{aligned}
d c^{(n)} / d t= & -\left(k_{d}+k_{g} m^{(0)}\right) c^{(n)}+\sum_{j=0}^{n}\left(\begin{array}{l}
n \\
j
\end{array}\right) \\
& \times c^{(j)} x_{m}^{n-j}\left[(-1)^{n-j} k_{d}+k_{g} m^{(0)}\right]+I x^{* n}
\end{aligned}
$$

and

$$
d m^{(0)} / d t=\left(k_{d}-k_{g} m^{(0)}\right) c^{(0)}-I x^{*} / x_{m} .
$$

For $n=0$ and 1 the first two moment equations for crystals are

$$
\begin{aligned}
& d c^{(0)} / d t=I, \\
& d c^{(1)} / d t=-x_{m}\left(k_{d}-k_{g} m^{(0)}\right) c^{(0)}+I x^{*} .
\end{aligned}
$$

Multiplying $d m^{(0)} / d t$ by $x_{m}$ gives monomer mass, and then Eqs. (2.13) and (2.15) satisfy the mass balance, $x_{m} d m^{(0)} / d t$ $=-d c^{(1)} / d t$. As time approaches infinity, the nucleation rate will vanish as the supersaturation approaches unity, and a thermodynamic equilibrium condition will finally be achieved. At equilibrium or steady state the derivative with respect to time equals zero, and by Eq. (2.13) or (2.15), the total concentration of polymer chains in solution becomes

$$
m_{\mathrm{eq}}^{(0)}=k_{d} / k_{g} \text {. }
$$

We define the dimensionless quantities,

$$
\begin{aligned}
& S=m^{(0)} / m_{\mathrm{eq}}^{(0)}, \quad C^{(n)}=c^{(n)} / m_{\mathrm{eq}}^{(0)} x_{m}^{n}, \quad \theta=t k_{g} m_{\mathrm{eq}}^{(0)}, \\
& J=I /\left(m_{\mathrm{eq}}^{(0)}\right)^{2} k_{g} .
\end{aligned}
$$

The moment equations can be written in dimensionless form,

$$
\begin{aligned}
& d S / d \theta=(1-S) C^{(0)}-\left(x^{*} / x_{m}\right) J, \\
& d C^{(0)} / d \theta=J, \\
& d C^{(1)} / d \theta=-(1-S) C^{(0)}+\left(x^{*} / x_{m}\right) J .
\end{aligned}
$$

Microscopic reversibility provides the thermodynamic equilibrium, $S_{\text {eq }}=1$, in Eq. (2.18), as $d S / d \theta=0$ and $J=0$ at the end of crystallization. For homogeneous nucleation, the initial conditions are $S(\theta=0)=S_{0}, C^{(0)}(\theta=0)=0, C^{(1)}(\theta=0)=0$, meaning that no preexisting nuclei are involved. The source term $J$ represents the nucleation rate of crystals of mass $x^{*}$.

The mass of a critical nucleus relative to the monomer mass depends solely on the interfacial energy and the supersaturation, ${ }^{20}$

$$
x * / x_{m}=(\omega / \ln S)^{d},
$$

where $d$ represents the dimension of the crystal structure and $\omega$ presents the ratio of interfacial energy to thermal energy, written as

$$
\omega=\left(4 \pi \rho / 3 x_{m}\right)^{1 / 3} 2 \sigma x_{m} / \rho k_{B} T
$$

for 3D spherical structures and

$$
\omega=2 \sigma\left(x_{m} L / \rho\right)^{1 / 2} / k_{B} T
$$

for 2D lamellar systems. The critical nucleus mass increases with time as supersaturation $S$ decreases. The scaled mass balance equation in a closed system follows from Eqs. (2.18) and (2.20),

$$
C^{(1)}(\theta)+S(\theta)=C_{0}^{(1)}+S_{0},
$$

where $C_{0}^{(1)}$ is the initial mass of crystals in polymer solution or melt, representing heterogeneous nucleation nuclei or seeds. For homogeneous nucleation, $C_{0}^{(1)}=0$. Based on Eq. 
(2.5), the homogeneous nucleation rate is written in dimensionless form as

$$
J=\alpha S^{2} \exp \left[-(d-1)^{-1} \omega^{d} /(\ln S)^{d-1}\right]
$$

with $\alpha=\left(2 \sigma x_{m} / \pi\right)^{1 / 2} / \rho k_{g}$. By Eq. (2.21), the number of monomers included in the critical nucleus, $x * / x_{m}$, is written in terms of supersaturation $S$, for the specific lamellar structure,

$$
x^{*} / x_{m}=\omega^{2} /(\ln S)^{2} .
$$

The substitution of the scaled nucleation rate yields the fully dimensionless equations for 2D lamellae system,

$$
\begin{aligned}
& d S / d \theta=(1-S) C^{(0)}-\alpha \omega^{2} S^{2} \exp \left(-\omega^{2} / \ln S\right) /(\ln S)^{2}, \\
& d C^{(0)} / d \theta=\alpha S^{2} \exp \left(-\omega^{2} / \ln S\right),
\end{aligned}
$$

and

$$
d C^{(1)} / d \theta=-(1-S) C^{(0)}+\alpha \omega^{2} S^{2} \exp \left(-\omega^{2} / \ln S\right) /(\ln S)^{2} .
$$

For 3D spherical crystal growth, however, the difference between the local-equilibrium interfacial concentration at the curved crystal surface, $m_{\mathrm{eq}}^{(0)}$, and the solubility of a flat surface, $m_{\infty}^{(0)}$, cannot be neglected. The Gibbs-Thomson factor $\Omega$ in Eq. (2.7) is written in term of crystal size $x / x_{m}$,

$$
\Omega=\omega /\left(x / x_{m}\right)^{1 / d},
$$

where $d$ is the dimension of the crystal structure and $\omega$ is the interfacial energy. Instead of being scaled by $m_{\text {eq }}^{(0)}$ as in 2D systems, the dimensionless quantities are redefined as

$$
\begin{aligned}
& S=m^{(0)} / m_{\infty}^{(0)}, \quad C^{(n)}=c^{(n)} /\left(m_{\infty}^{(0)} x_{m}^{n}\right), \quad \theta=t k_{g} m_{\infty}^{(0)}, \\
& J=I /\left(m_{\infty}^{(0)}\right)^{2} k_{g} .
\end{aligned}
$$

Equation (2.13)-(2.15) are moment equations, so the single crystal size $x / x_{m}$ is approximated by average size of crystal $C^{\text {avg }}$. Thus Eqs. (2.13)-(2.15) are scaled in the form

$$
\begin{aligned}
d S / d \theta= & \left(-S+e^{\Omega a}\right) C^{(0)}-\alpha \omega^{3} S^{2} \\
& \times \exp \left[-\omega^{3} / 2(\ln S)^{2}\right] /(\ln S)^{3}, \\
d C^{(0)} / d \theta= & \alpha S^{2} \exp \left[-\omega^{3} / 2(\ln S)^{2}\right],
\end{aligned}
$$

and

$$
\begin{aligned}
d C^{(1)} / d \theta= & -\left(-S+e^{\Omega a}\right) C^{(0)}+\alpha \omega^{3} S^{2} \\
& \times \exp \left[-\omega^{3} / 2(\ln S)^{2}\right] /(\ln S)^{3},
\end{aligned}
$$

where $\Omega_{a}=\omega /\left(C^{\mathrm{avg}}\right)^{1 / 3}$ represents the average GibbsThomson effect.

The crystallinity is defined as the ratio of the mass crystallized at time $t$ divided by the total mass crystallized,

$$
X=\left(C^{(1)}-C_{0}^{(1)}\right) /\left(C_{\mathrm{eq}}^{(1)}-C_{0}^{(1)}\right) .
$$

The ordinary differential moment equations are readily solved by standard software.

\section{B. Numerical methods}

The growth and dissociation rate coefficients are assumed constant in the above moment method, but more generally, the rate coefficients are power law expressions for the mass dependence. ${ }^{20}$ For crystal growth, the rate coefficient may be written as

$$
k_{g}(x)=\kappa_{g} x^{\lambda},
$$

where $\kappa_{g}$ is a prefactor whose units are determined by the power $\lambda$. The dissociation rate is determined by applying microscopic reversibility for the growth process,

$$
k_{d}(x)=m_{\mathrm{eq}}^{(0)} k_{g}(x) .
$$

The exponent $\lambda$ equal to $0,1 / 3$, and $2 / 3$ represents surfaceindependent, diffusion-controlled, and surface-controlled deposition rates, respectively. ${ }^{20}$

We define dimensionless quantities ${ }^{21}$ consistent with Eq. (2.17),

$$
\begin{aligned}
& \xi=x / x_{m}, \quad \theta=t \kappa_{g} m_{\infty}^{(0)} x_{m}^{\lambda}, \quad S=m^{(0)} / m_{\infty}^{(0)}, \\
& C=c x_{m} / m_{\infty}^{(0)}, \quad C^{(n)}=c^{(n)} / m_{\infty}^{(0)} x_{m}^{n}, \quad J=I / \kappa_{g} m_{\infty}^{(0)} x_{m}^{\lambda},
\end{aligned}
$$

and note that $\xi$ is the number of monomers in a crystal. The time $\theta$, crystal size distribution $C(\xi, \theta)$, and monomer concentration $S(\theta)$ are scaled by the equilibrium monomer concentration $m_{\infty}^{(0)}$. Substitution of Eq. (2.38) into Eqs. (2.10) and (2.11) yields population balance equations in dimensionless form,

$$
d S(\theta) / d \theta=\left[-S(\theta)+e^{\Omega a}\right] C^{(\lambda)}+J \xi^{*}
$$

and

$$
\begin{aligned}
\partial C(\xi, \theta) / \partial \theta= & S(\theta)\left[-\xi^{\lambda} C(\xi, \theta)+(\xi-1)^{\lambda} C(\xi-1, \theta)\right] \\
& -\exp [\Omega(\xi)] \xi^{\lambda} C(\xi, \theta)+\exp [\Omega(\xi+1)] \\
& \times(\xi+1)^{\lambda} C(\xi+1, \theta)-J \delta\left(\xi-\xi^{*}\right),
\end{aligned}
$$

where $\Omega(\xi)$ is related to the crystal dimension $d,{ }^{20}$

$$
\Omega(\xi)=\omega / \xi^{1 / d} .
$$

Since Eq. (2.39) is a moment equation, $\Omega_{a}$ is related to the average number of monomers in the crystal $C^{\text {avg }}$,

$$
\Omega_{a}=\omega /\left(C^{\mathrm{avg}}\right)^{1 / d} .
$$

We note that moment equations cannot be derived because of $\xi$ in the exponential term. Thus, moment methods are not applicable for $\lambda>0$ and numerical schemes have to be employed to solve the equations.

\section{Heterogeneous nucleation}

To promote nucleation in supersaturated liquid or glass, small impurity (second phase) particles are often introduced deliberately. These impurity particles, acting as nucleation seeds, grow by depositing monomer on their surface. The activation energy for homogeneous nucleation presents a significant barrier for stable nuclei to be formed, whereas heterogeneous nucleation is limited only by monomer diffusion to the solid surfaces. For these ideal conditions, homogeneous nucleation would be negligible and heterogeneous 




(a)


(c)



FIG. 1. Time evolution of $S, C^{(0)}$, $C^{\text {avg }}$, and $X$ as $\alpha$ varies among $10^{-1}, 10^{-2}$, $10^{-3}$, and $10^{-4}$ with $\omega=5, \lambda=0$.

nucleation dominant, the case we now consider. For heterogeneous nucleation, we set $I=0$, thus the growth rate of the number of crystals, $d C^{(0)} / d \theta$, equals zero, and the population balance equations reduce to a single ordinary differential equation. For the case of $\Omega=0$ (flat surface),

$$
d S / d \theta=(1-S) C_{0}^{(0)},
$$

where $C_{0}^{(0)}$ is the number of nucleation agents. The exact solution, given the initial condition $S(\theta=0)=S_{0}$, is written as

$$
S=1+\left(S_{0}-1\right) \exp \left(-C_{0}^{(0)} \theta\right) .
$$

Consistent with the crystallinity definition, Eq. (2.35), and mass conservation, Eq. (2.24), the crystallinity for heteroge- neous nucleation is expressed in terms of supersaturation $S$ and scaled time $\theta$,

$$
X=\left[S_{0}-S(\theta)\right] /\left(S_{0}-S_{\mathrm{eq}}\right) .
$$

Substitution of Eq. (2.44) into Eq. (2.45) results directly in the crystallinity versus time evolution equation,

$$
X=1-\exp \left(-C_{0}^{(0)} \theta\right),
$$

which is the Avrami equation with growth rate $K=c_{0}^{(0)} k_{g}$ and Avrami exponent $n=1$.

\section{RESULTS AND DISCUSSION}

The flat growth surface of lamellar crystal simplifies polymer nucleation and growth into readily solved moment equations by reducing the Gibbs-Thomson effects. These moment differential equations, Eqs. (2.27)-(2.29), are solved by NDSOLVE in Mathematica ${ }^{\circledR}$ for various values of the parameters. The parameter $\omega$ represents the ratio of interfacial energy to thermal energy (Eq. (2.22)) and, based on published values for the interfacial energy, ${ }^{22}$ is chosen to span two orders of magnitude, $0.1-10$. The nucleation rate prefactor $\alpha$, chosen to span widely from 0.0001 to 100 , depends on the combination of the liquid-solid interfacial energy $\sigma$, monomer molecular mass $x_{m}$, solid phase density $\rho$, and growth rate coefficient $k_{g}$. For homogeneous nucleation, the initial source term $C_{0}^{(0)}$ is set to zero. An initial condition of $S_{0}=50$ is chosen to minimize the effects of denucleation in the computation.

Figure 1 presents the time dependence of the key variables in polymer crystallization, as computed via distribution kinetics. The time evolutions of supersaturation $S$ [Fig. 1(a)], number of crystals $C^{(0)}$ [Fig. 1(b)], the average number of crystallized monomers $C^{\text {avg }}$ [Fig. 1(c)], and the degree of crystallinity $X[$ Fig. 1(d)] are shown at various values of $\alpha$ for the 2D system. A typical $S$-shape curve of polymer crystallization is confirmed in Fig. 1(a). As the prefactor $\alpha$ increases, the overall crystallization rate increases, which is shown by the time needed to reach the steady state. A large $\alpha$ also leads to a large number of crystals at equilibrium [Fig. 1(b)]. The average number of monomers in the crystal at equilibrium $C^{\text {avg }}$ decreases as $\alpha$ rises [Fig. 1(c)], since large $\alpha$ means a greater nucleation rate and results in a larger number of crystals at equilibrium. The prefactor $\alpha$ also has a negative influence on the induction time of crystallization because a large initial nucleation rate will shorten the induction time. The crystallinity time dependence [Fig. 1(d)] is a mirror image of the supersaturation time evolution [Fig.

TABLE I. Effect of $\alpha$ on Avrami exponent $n$ for $\lambda=0, \omega=5, S_{0}=50$, and $C_{0}^{(0)}=0$.

\begin{tabular}{ccc}
\hline \hline$\alpha$ & $n(2 \mathrm{D})$ & $n(3 \mathrm{D})$ \\
\hline $10^{-4}$ & 2.20 & 1.00 \\
$10^{-2}$ & 2.17 & 1.23 \\
$10^{-1}$ & 2.10 & 1.46 \\
$10^{0}$ & 1.44 & 1.00 \\
$10^{2}$ & 1.00 & 1.00 \\
\hline
\end{tabular}


TABLE II. Effect of $\omega$ on Avrami exponent $n$ for $\alpha=0.1, \lambda=0, S_{0}=50$, and $C_{0}^{(0)}=0$.

\begin{tabular}{ccl}
\hline \hline$\omega$ & $n(2 \mathrm{D})$ & $n(3 \mathrm{D})$ \\
\hline 0.1 & 1.97 & 1.9 \\
4.0 & 1.80 & 1.48 \\
5.0 & 1.77 & 1.46 \\
6.0 & 1.76 & 1.35 \\
7.0 & 1.75 & 1.12 \\
10 & 1.75 & $\ldots$ \\
\hline \hline
\end{tabular}

1(a)]. Following a $S$-shape curve, as observed in experiments, the crystallinity evolves to unity as supersaturation decreases to the equilibrium state.

Because the plotted experimental data and simulations are not strictly straight lines, a defined method is needed to determine the slopes. The straight part of most plots begins at $X=0.1$ and ends at $X=0.9$, and includes the most significant range of data. We therefore used points corresponding to this interval in the measurement of slopes reported in Tables I-III.

The effects of $\alpha$ on the Avrami exponent are compared for $2 \mathrm{D}$ and $3 \mathrm{D}$ systems in Fig. 2. The interfacial energy $\omega$ is set to 5, a surface-independent growth and dissociation rates is proposed $(\lambda=0)$, and the prefactor $\alpha$ is chosen to span widely from $10^{-4}$ to $10^{2}$. According to Eq. (1.6), the Avrami exponent $n$ is the slope of the double logarithm plot of $-\ln (1-X)$ versus scaled time $\theta$. Figure 2 presents the Avrami plots for 2D and 3D systems as $\alpha$ varies from 0.0001 to 100 . In contrast to the Avrami equation, these plots are not strictly straight lines, but curve slightly up at the beginning of crystallization and down at the final stage of crystallization. Curving up at the beginning is caused by the induction time, and the final curving down shows the approach to saturation. Hay $^{13}$ reported that the Avrami equation provided a poor approximation at the final stage of crystallization because experimental data deviated from the straight line by curving down. We conclude that the distribution kinetics model, by accurately predicting this behavior, more realistically represents the curve.

In the 2D system, an apparent slope difference of the Avrami plots is observed. The slope value for each plot is measured and tabulated in Table I. We note the slope increases from 1.00 at $\alpha=10^{2}$ to 2.20 at $\alpha=10^{-4}$. However, when $\alpha$ is less than $10^{-4}$, the lines move horizontally right and the slope variation is too small to be measured. All plots collapse into one straight line when $\alpha$ is greater than $10^{2}$. In 3D a smaller slope difference is observed [Fig. 2(b)]. The

TABLE III. Effect of $\lambda$ on Avrami exponent $n$ for $\alpha=0.1, \omega=5, S_{0}=50$, $C_{0}^{(0)}=0.0001$, and $C_{0}^{(1)}=0$.

\begin{tabular}{ccc}
\hline \hline$\lambda$ & $n(2 \mathrm{D})$ & $n(3 \mathrm{D})$ \\
\hline 0 & 1.70 & 1.44 \\
$1 / 3$ & 2.00 & 1.64 \\
$2 / 3$ & 3.09 & 2.57 \\
0.93 & 5.27 & 4.29 \\
0.98 & 5.32 & 4.50 \\
\hline
\end{tabular}


FIG. 2. The effects of $\alpha$ on (a) 2D and (b) 3D crystallinity plots with $\omega$ $=5, \lambda=0, S_{0}=50$, and $C_{0}^{(0)}=0$.

slope increases as $\alpha$ varies from $10^{-4}$ to 0.1 , and drops down to 1.00 as $\alpha$ increases to 1 . When $\alpha$ is greater than unity or less than $10^{-4}$, no measurable slope change. All the plots with $\alpha$ greater than 1.0 collapse into one straight line and all the plots with $\alpha$ less than $10^{-4}$ are only transposed horizontally.

The ratio of interfacial to thermal energy, $\omega$ influences nucleation and growth. By moment computations, the effects of $\omega$ are investigated for the 2D and 3D systems (Fig. 3). Figure 4 shows results of numerical computations for $\omega$ equal to 4,5 , and 6 . The dotted lines represent 2D while the solid lines represent the 3D solution. The slopes for Figs. 3


FIG. 3. The effects of $\omega$ on (a) 2D and (b) 3D crystallinity plots by a moment solution with $\alpha=0.1, \lambda=0, S_{0}=50$, and $C_{0}^{(0)}=0$. 


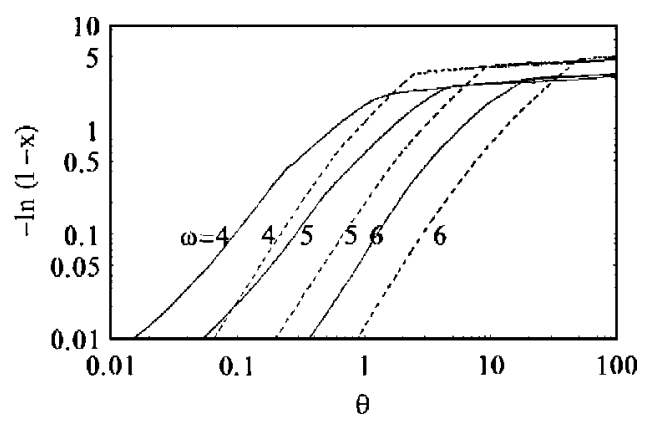

FIG. 4. The comparison of crystallinity plots by numerical solution for $2 \mathrm{D}$ (dotted line) and 3D (solid line) with $\lambda=0, \alpha=0.1$, and $S_{0}=50$.

and 4 are reported in Table II. The slope variation as $\omega$ changes is quite small in both $2 \mathrm{D}$ and $3 \mathrm{D}$, and a larger slope is observed in the $2 \mathrm{D}$ case. According to Eq. (2.26), a small value of $\omega$ leads to a small critical size of crystal at constant supersaturation, and finally leads to a large nucleation rate. Increasing $\omega$ delays nucleation and the decrease of supersaturation. Figure 3(a) presents the double logarithm plots as $\omega$ varies among $0.1,4,7$, and 10 for the 2D system. Different slopes, ranging from 1.75 at $\omega=10$ to 1.97 at $\omega=0.1$, are observed (Table II). Similar to the effect of the nucleation prefactor $\alpha$, the influence of interfacial energy is notable only if $\omega$ is small. The slope difference disappears when $\omega$ is large, e.g., the slope at $\omega=7$ is almost same as at $\omega=10$. A reasonable explanation is that the crystal growth becomes the dominant term if $\omega$ is large, since the nucleation term exponentially decreases with $\omega^{2}$ as shown in Eq. (2.25). In the 3D system, a more noticeable slope variation is observed at different $\omega$. The slope varies from 1.90 to 1.12 as $\omega$ changes from 0.1 to 7 . The explanation for the greater influence of $\omega$ in the $3 \mathrm{D}$ system, according to Eq. (2.25), is that the nucleation rate is a function of $\omega^{3}$ in $3 \mathrm{D}$ and of $\omega^{2}$ in 2D. Comparing the numerical and the moment results (Figs. 3 and 4, respectively) reveals that the numerical result of crystallinity reaches an asymptotic value at large time while the moment result continues to increase. This is the influence of denucleation, which is ignored in the moment computations.

Different values of $\alpha$ and $\omega$ have the expected effects as shown in Fig. 5, larger values of $\alpha$ shift the curves to smaller times, whereas larger values of $\omega$ give smaller times. These findings for 2D are similar to 3D results. Figure 6 shows that the effect of increasing the initial supersaturation is to shift



FIG. 5. The effects of different values of $\alpha$ and $\omega$ on 2D crystallinity with $\lambda=0, S_{0}=50$, and $C_{0}^{(0)}=0 ; \mathrm{I}-\alpha=100, \omega=0.001 ; \mathrm{II}-\alpha=0.001, \omega=0.001$; III $-\alpha=100, \omega=10 ;$ IV $-\alpha=0.001, \omega=10$.

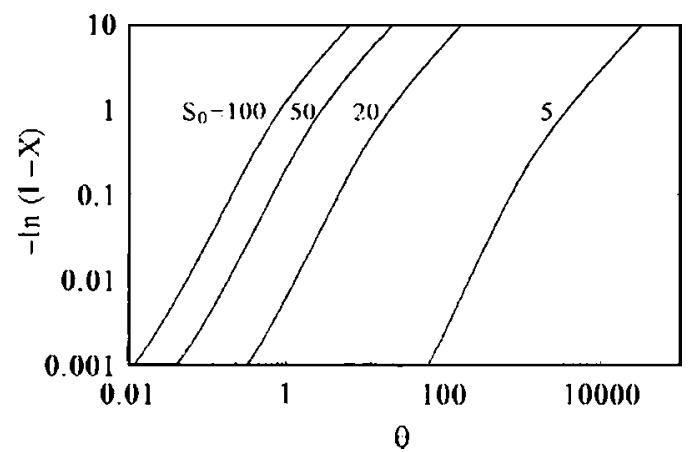

FIG. 6. The effect of initial supersaturation $S_{0}$ on 2D crystallinity with $\alpha$ $=0.1, \omega=5, \lambda=0$, and $C_{0}^{(0)}=0$.

the Avrami curves to smaller times. Changing $S_{0}$ has little influence on the slope, which increases from 1.69 to 1.78 when $S_{0}$ increases from 5 to 100 .

The exponent of growth and dissociation rates $\lambda$ is 0 , $1 / 3$, and $2 / 3$, for surface-independent, diffusion-controlled, and surface-controlled deposition rate, respectively (Fig. 7). To explore more thoroughly the effect of $\lambda$, we included $\lambda$ $=0.93$ and 0.98 in Table III. A possible explanation for the larger $\lambda(>2 / 3)$ is the increasing mass dependence of deposition rate caused by shear force during fluid movement or by microscopic structural changes. ${ }^{23}$ Equations (2.39) and (2.40) were solved at the different values of $\lambda$ by a numerical procedure described previously. ${ }^{20}$ According to Eqs. (2.39) and (2.42), a nonzero initial condition of $C_{0}^{(0)}$ should be chosen to avoid singularities near $t=0$ in the numerical computation. In our simulation, $S_{0}=50, C_{0}^{(0)}=0.0001$, and $C_{0}^{(1)}=0$ are the initial conditions. Figures 7 (a) and 7 (b) present the effects of $\lambda$ on 2D and 3D systems, respectively. Different


FIG. 7. The effects of $\lambda$ with $\alpha=0.1, \omega=5, S_{0}=50, C_{0}^{(0)}=0.01$, and $C_{0}^{(1)}=1$ for (a) $2 \mathrm{D}$ and (b) 3D. 

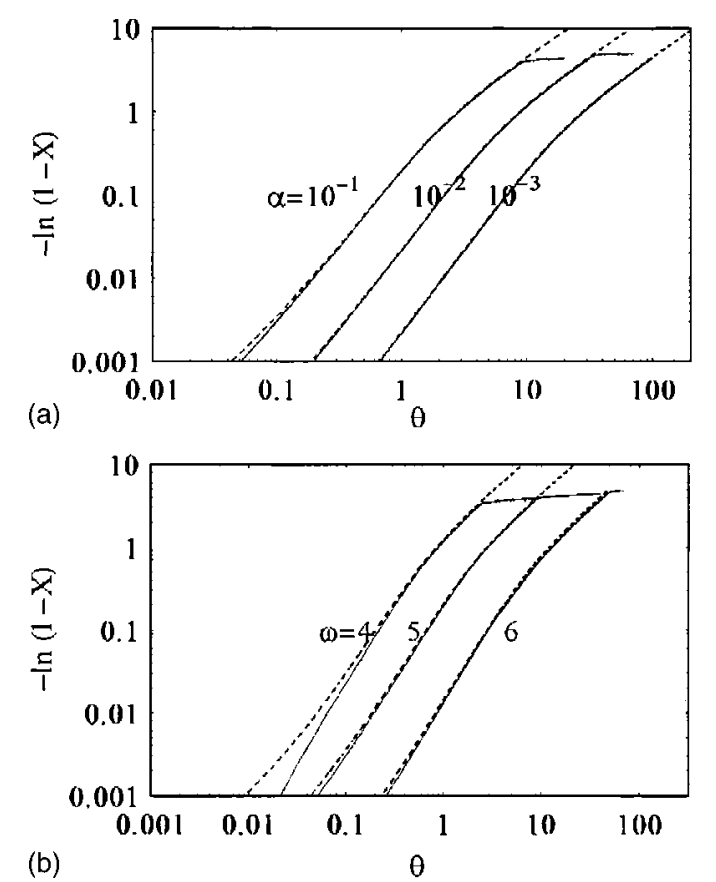

FIG. 8. The comparison of moment method (dashed line) with numerical method (solid line) for: (a) $\alpha$ equal to $0.1,0.01$, and 0.001 with $\omega=5, \lambda$ $=0, S_{0}=50$, and $C_{0}^{(0)}=0$; (b) $\omega$ equal to 4,5 , and 6 with $\alpha=0.1, \lambda=0, S_{0}$ $=50$, and $C_{0}^{(0)}=0$.

slopes are confirmed as $\lambda$ varies in both $2 \mathrm{D}$ and $3 \mathrm{D}$ cases, as shown in Table III. The range of slope values is consistent with reported experimental measurements ${ }^{24}$ for the Avrami exponent $n$ in Eq. (1.6), $1 \leqslant n \leqslant 4$. Avrami exponents greater than 4 are occasionally reported; for example, slopes of up to 5.0 for syndiotactic polystyrene crystallization were found by Yoshioka and Tashiro, ${ }^{23}$ who suggested conelike spherulite growth as a potential explanation for the large value of $n$.

The influence of geometry dimension is also confirmed by comparing the slopes for 2D and 3D systems. Smaller slopes are found in the 3D system, as shown by Tables I-III. The parametric effects are also different for 2D and 3D systems. We note that $\omega$ has less effect on the Avrami exponent in the 2D system, whereas $\alpha$ has a larger effect. Compared with the effects of the other parameters, $\lambda$ has a substantial influence on the Avrami exponent.

A comparison of moment methods and numerical methods is made for the 2D system to investigate the effects of denucleation (Fig. 8). Figure 8(a) presents the comparison of moment and numerical solutions as $\alpha$ varies. Figure 8(b) shows the comparison of these two solutions, both for flat growth surfaces, at different $\omega$. The dotted line presents the moment simulation and the solid line is the numerical solution. Although the two solutions are consistent at the beginning of crystallization, an increasing discrepancy is observed near the end of crystallization, where crystallinity $X$ is about 0.99. This discrepancy caused by the increasing effect of denucleation that can only be computed numerically. Denucleation, the reverse process of nucleation, results from the stability shift of formed crystals from stable to unstable. The reduction of supersaturation during crystallization, according to Eq. (2.26), increases the nucleus critical size. As the supersaturation decreases, nuclei smaller than the critical size



FIG. 9. The comparison of experimental data of nylon- 6 and moment solution: $(\boldsymbol{\square}), T=188^{\circ} \mathrm{C} ;(\boldsymbol{\Delta}), T=190^{\circ} \mathrm{C} ;(\boldsymbol{\bullet}), T=192{ }^{\circ} \mathrm{C}$.

become unstable and dissolve instantaneously, ${ }^{20}$ while nuclei larger than the critical size keep growing. At the beginning of crystallization when the supersaturation is large, the denucleation rate, compared with nucleation rate, is too small to have a noticeable effect on the time evolution of degree of crystallinity. ${ }^{20}$ As the crystal keeps growing, however, more and more nuclei become unstable and tend to dissolve because of the increasing critical size of nucleus. At the end of crystallization, the effect of denucleation, compared with the nucleation rate, can become substantial, and is manifested as Ostwald ripening.

The validity of the distribution kinetics model is also examined by comparison with experimental data (Fig. 9). The points are experimental data ${ }^{25}$ for nylon- 6 based on real time $t(\mathrm{~min})$ at $T=188,190$, and $192{ }^{\circ} \mathrm{C}$. The initial supersaturation $S_{0}$ has not been reported for the experiments and is assumed to be 50 in the computations. To compare with the model based on dimensionless time $\theta=t k_{g} m_{\mathrm{eq}}^{(0)}$, a transposition of the simulation results is applied. According to the definition of dimensionless time, Eq. (2.17), a distance of $\ln \left(k_{g} m_{\mathrm{eq}}^{(0)}\right)$ units is transposed horizontally to the left to convert the simulation results into plots based on real time $t(\min )$. A zero horizontal distance is transposed to fit the experimental data at $T=188{ }^{\circ} \mathrm{C}$, thus $k_{g} m_{\mathrm{eq}}^{(0)}=1.00 \mathrm{~min}^{-1}$. Similarly, the values of $k_{g} m_{\mathrm{eq}}^{(0)}$ at $T=190$ and $192{ }^{\circ} \mathrm{C}$ are readily determined by the measurements of the horizontal transposition distance to be 0.80 and $0.68 \mathrm{~min}^{-1}$, respectively. The experimental measurements at $T=190$ and $192{ }^{\circ} \mathrm{C}$ are horizontal transpositions of the simulation results at $T=188^{\circ} \mathrm{C}$, and there is no slope variation. This is consistent with the understanding that $k_{g} m_{\mathrm{eq}}^{(0)}$ depends on temperature.

Figure 10 presents an Avrami plot for experimental polypropylene (PP) data at $110{ }^{\circ} \mathrm{C} .^{7}$ The scattered points are the measurements, the solid line is a fit of the distribution kinetics model, and the dashed line is the Avrami equation with $n=3.0 .^{7}$ Figure 10 (a) shows the evolution of crystallinity $X$ versus real time. The Avrami equation with $n=3.0$ fits the data fairly well except where the data curve down and deviate from the Avrami equation at the end of crystallization [Fig. 10(b)]. The solid line is our model prediction for $\lambda$ $=2 / 3, \alpha=0.1$, and $\omega=5$. The predicted slope is 3.09, as reported in Table III, and is close to the value 3.0 reported by Ryan. ${ }^{7}$ The scaling factor for time is $\kappa_{g} m_{\infty}^{(0)} x_{m}^{\lambda}=6.76$ 



FIG. 10. The fit of the model to experimental data of polypropylene (Ref. 7); the solid line is a fit of the model and the dashed line is the Avrami equation with $n=3.0$.

$\times 10^{-3} \mathrm{~min}^{-1}$. It is interesting that the curving down at the end of crystallization is predicted in the crystal size distribution model and fits the experimental data quite well. The Avrami equation, by contrast, provides a constant slope, and thus fits only the intermediate data.

We also compared the Avrami exponent determined in our theory with published experimental measurements. According to Tables I-III, for $\lambda \leqslant 2 / 3$, the model shows a range of 1-5 for the Avrami exponent, consistent with most published values. ${ }^{26-28}$

For heterogeneous nucleation, the distribution kinetics directly results in an Avrami equation with growth rate $K$ $=c_{0}^{(0)} k_{g}$ and Avrami exponent $n=1$, as suggested in Eq. (2.46). The double logarithm plots are made to investigate the effect of $C_{0}^{(0)}$. It is confirmed that the crystallization rate increases with the number of nucleation agents, as shown in Fig. 11. The Avrami exponent, which is the slope of the double logarithm plot, always equals unity for $\lambda=0$. It is possible, however, that homogeneous and heterogeneous nucleation occur simultaneously, yielding $n>1$.



FIG. 11. The Avrami plot as $C_{0}^{(0)}$ varies from 0.01 to 0.03 in steps of 0.01 for heterogeneous nucleation with $\lambda=0$ and $S_{0}=50$.



FIG. 12. The effect of $\lambda$ for heterogeneous nucleation at $C_{0}^{(0)}=0.01, C_{0}^{\text {avg }}$ $=75, S_{0}=50$, and $\omega=5$.

The effect of $\lambda$ on the Avrami exponent is also investigated for heterogeneous nucleation, as shown in Fig. 12. It is observed that the overall crystallization rate increases as $\lambda$. The equilibrium crystallinity is reached at $\theta=10,100$, and 1000 at $\lambda=2 / 3,1 / 3$, and 0 , respectively. The Avrami exponent $n$ also increases with $\lambda$, predicting values 1.76 at $\lambda$ $=2 / 3,1.31$ at $\lambda=1 / 3$, and 1.00 at $\lambda=0$. Compared with $n$ for homogeneous nucleation in Table III, the $n$ values for heterogeneous nucleation are small. This is explained by the additional kinetics contribution caused by the increase of the number of nuclei in homogeneous nucleation, which does not arise in heterogeneous nucleation because the number of nuclei is constant. We also note that the slope variation is smaller than in homogeneous nucleation, because the additional kinetics contribution in homogeneous nucleation increases as $\lambda$.

\section{CONCLUSION}

Nucleation and crystal growth are essential phenomena in quantitatively describing the evolution of a crystallizing polymer solution or melt. A kinetics model based on cluster distribution dynamics incorporates these processes and realistically represents the time evolution of crystallinity. The model includes rate coefficients for crystal growth $k_{g}$ and crystal dissociation $k_{d}$. Based on widely accepted notions, a 2D lamellar structure for the polymer crystal nucleus is proposed, and thus the Gibbs-Thomson effect is excluded for the 2D lamellar structure system. A 3D spherical structure is also investigated to demonstrate the influence of GibbsThomson effects. Population balance equations based on crystal and amorphous polymer segments lead to the dynamic moment equations for the molar concentrations for mass independent monomer deposition rate coefficients. $\mathrm{Nu}-$ merical solution is required if the deposition rate is diffusion or surface controlled and the rate coefficients are consequently size-dependent power expressions.

Although it is widely agreed that the Gibbs-Thomson effect is critical for understanding nucleation and crystal growth, less acknowledged is that the Gibbs-Thomson effects can be neglected for the flat growth surface of a specific lamellar structure. Our proposal is that the combined processes of nucleation and crystal growth can be described by moment equations developed from distribution kinetics, i.e., population dynamics theory. The validity of moment methods is examined by comparison with the numerical methods. 
Consistency is confirmed between these two methods except for the discrepancy at the end of crystallization caused by denucleation.

Another goal of our current work has been to reconcile distribution kinetics and the empirical Avrami equation by examining the detailed, fundamental features of nucleation mechanism and crystal growth. The comparison with general experimental observations suggests that distribution kinetics is a more realistic approximation at the end of crystallization than the Avrami transition theory. The investigation of model parameters offers a quantitative way to determine Avrami parameters, which can only be determined empirically by Avrami transition theory.

${ }^{1}$ A. Keller, Philos. Mag. 2, 1171 (1957).

${ }^{2}$ L. H. Sperling, Introduction to Physical Polymer Science, 2nd ed. (WileyInterscience, New York, 1992).

${ }^{3}$ C. M. Chen and P. G. Higg, J. Chem. Phys. 108, 4305 (1998).

${ }^{4}$ J. K. Doye and D. Frenkel, J. Chem. Phys. 110, 2692 (1999).

${ }^{5}$ L. Mandelken, Crystallization of Polymers, 2nd ed. (Cambridge University Press, Cambridge, 2001).

${ }^{6}$ R. A. Shanks and L. Yu, Polymer Material Encyclopedia (CRC, Boca Raton, FL, 1996).

${ }^{7}$ A. J. Ryan, J. A. Fairclough, N. J. Terrill, P. D. Olmsted, and W. D. Poon,
Faraday Discuss. 112, 13 (1999).

${ }^{8}$ F. C. Frank and M. Tosi, Proc. R. Soc. London, Ser. A 263, 323 (1961).

${ }^{9}$ J. K. Doye and D. Frenkel, J. Chem. Phys. 109, 10033 (1998).

${ }^{10}$ M. Avrami, J. Chem. Phys. 7, 1103 (1939).

${ }^{11}$ U. R. Evans, Trans. Faraday Soc. 41, 365 (1945).

${ }^{12}$ P. Meares, Polymers: Structure and Properties (Van Nostrand, New York, 1965).

${ }^{13}$ J. N. Hay, Br. Polym. J. 3, 74 (1971).

${ }^{14}$ M. Avrami, J. Chem. Phys. 8, 212 (1940).

${ }^{15}$ Y. Long, R. A. Shanks, and Z. H. Stachurski, Prog. Polym. Sci. 20, 651 (1995).

${ }^{16}$ B. J. McCoy, Ind. Eng. Chem. Res. 40, 5147 (2001).

${ }^{17}$ G. Madras and B. J. McCoy, Chem. Eng. Sci. 59, 2753 (2004).

${ }^{18}$ M. Avrami, J. Chem. Phys. 9, 177 (1941).

${ }^{19}$ R. B. McClurg and R. C. Flagan, J. Colloid Interface Sci. 228, 194 (1998).

${ }^{20}$ G. Madras and B. J. McCoy, Phys. Chem. Chem. Phys. 5, 5459 (2003).

${ }^{21}$ G. Madras and B. J. McCoy, J. Chem. Phys. 117, 6607 (2002).

${ }^{22}$ N. B. Singh and M. E. Glicksman, J. Cryst. Growth 98, 573 (1989).

${ }^{23}$ A. Yoshioka and K. Tashiro, Polymer 44, 6681 (2003).

${ }^{24}$ K. Nagarajan, K. Levon, and A. S. Myerson, J. Therm Anal. Calorim. 59, 497 (2000).

${ }^{25}$ W. Weng, G. H. Chen, and D. Wu, Polymer 44, 8119 (2003).

${ }^{26}$ Z. Qiu, S. Fujinami, M. Komura, M. Komura, K. Nakajima, T. Ikehara, and T. Nishi, Polymer 45, 4515 (2004).

${ }^{27}$ S. Kuo, W. Huang, S. Huang, H. Kao, and F. Chang, Polymer 44, 7709 (2003).

${ }^{28}$ K. Kajaks and A. Flores, Polymer 41, 7769 (2000). 